\title{
STUDY OF VARIOUS INTERNET OF THINGS PlatForms
}

\author{
Bhumi Nakhuva and Prof. Tushar Champaneria \\ Department of Computer Science and Engineering, L. D. College of Engineering, \\ Ahmedabad, Gujarat, India
}

\begin{abstract}
Internet of Things is a technological revolution which provides the vision of connected world of Things. Devices, people and systems are connected with one another in such a way that they can communicate with each other over Internet. So the form of communication from human-human is now turned into humanhuman, human-things and things- things. Variety of platforms are now a days available that can support entire development to deployment of IoT applications and systems. In this paper, we will first start with the introduction of IoT platforms as well as IoT middleware and then will discuss various IoT platforms, compare various IoT platforms and will end up with the conclusion.
\end{abstract}

\section{KEYWORDS}

Internet of Things, platform, middleware, development, cloud.

\section{INTRODUCTION}

"Internet of Things" (called IoT), associated with the concept of "future internet" is a vision where each object will become a part of the Internet. Here objects can be any living entity like humans or animals and any non-living entities on earth. IoT is like a vision in which every object which is on network can uniquely be identified, its status and position can be known, it is accessible to the network and also services and intelligences are added to this network. So it fuses real world with virtual world of digital technology and impacts our social, personal as well as professional life. IoT will change the information world and technology world drastically to make a more comfortable world full of technology for us. Various prototyping hardware boards, on chip systems, sensors, RFID and ubiquitous networking capabilities are supporting candidates for IoT evolution [1].

In this section, IoT platform and IoT middleware are elaborated. In section-II, various popular IoT platforms are discussed and then they are compared in section-III. At last the paper is concluded in section-IV.

\subsection{Internet of Things platform}

Platform: When you are developing some application, Platform is one which allows you to deploy and run your application. A platform could be a hardware plus software suite upon which other applications can operate. Platform could comprise hardware above which Operating system can reside. This Operating system will allow application to work above it by providing necessary execution environment to it. [3] 
IoT platforms (more specifically IoT application platforms) provide a comprehensive set of generic, i.e. application independent functionalities which can be used to build IoT applications. [4] When there is only one communication link between devices of one type with another device of same type then, a system of specific service can be set up. But in case of communication among devices of multiple types, there is a need of some common standard application platform which hides heterogeneity of various devices by providing a common working environment to them. [5] An IoT application platform is a virtual solution, means it resides over cloud. Data is the entity that drives business intelligence and every device has something to talk with other device that is data. By means of cloud connectivity, IoT application platform translates such devices data into useful information. So it provides user means to implement business use cases and enables predictive maintenance, pay-per-use, analytics and real time data management. Thus, IoT application platforms provide a complete suite for application development to its deployment and maintenance.

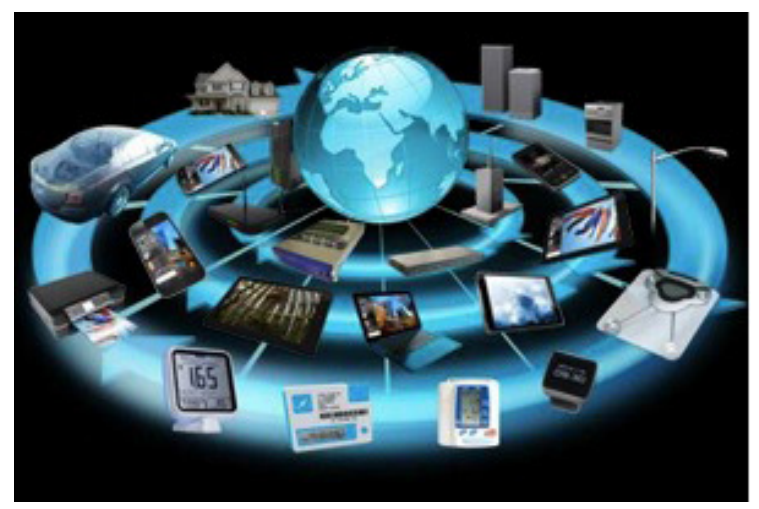

Figure 1. Internet of Things [2]

\subsection{Internet of Things middleware}

Middleware: Middleware provides a wide variety of services to the applications from outside. So the application is not bound to use its all services but only uses the necessary set of services. It acts as a middle level agent between the service provider and service consumer. It allows communication among the application that come from different vendors or runs on different platform. It acts as a mediator between applications of different forms. [3]

IoT middleware is a mechanism that joints different components of IoT systems together and offers smooth communication among devices and components. It is an interface that is available to provide interaction between Internet and Things. Here things may be hardware or some application. [6] IoT middleware aims to hide the heterogeneity of different hardware and software and enables the use of common platform so that the system is easy to use and manage. Thus, IoT middleware is a mediator suite which hides heterogeneity among devices, components and technology of an IoT system.

\subsection{Internet of Things platform Vs. Internet of Things middleware}

IoT platform is a complete suite of services that facilitates services like development, deployment, maintenance, analytics as well as intelligent decision making capabilities to an IoT application. 
Whereas IoT middleware is a service suite which is mainly intended to overcome the heterogeneity problem of the entire IoT system by enabling smooth communication among devices and components of different vendors and different technology.

\section{VARIOUS INTERNET OF THINGS PLATFORMS}

Various IoT platforms are now a day available that can be used for developing an IoT solution but in this section we have covered eleven popular platforms that are widely used for IoT solution building.

\subsection{Google Cloud Platform}

Developers can code, test and deploy their applications with highly scalable and reliable infrastructure that is provided by Google and Google itself uses it. Developers have to just pay attention to the code and Google handles issues regarding infrastructure, computing power and data storage facility. [7] Google is one of the popular IoT platform because of: Fast global network, Google's BigData tool, Pay as you use strategy, Support of various available services of cloud like RiptideIO, BigQuery, Firebase, PubSub, Telit Wireless Solutions, Connecting Arduino and Firebase and Cassandra on Google Cloud Platform and many more.

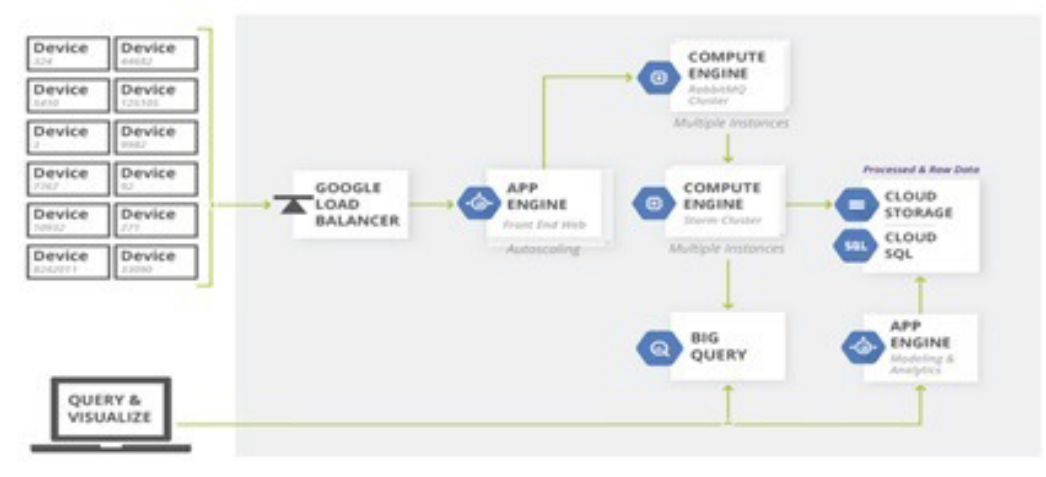

Figure 2. Real Time Stream Processing- Google IoT [8]

Figure-2 shows real time stream processing by Google. Devices send their status information to App Engine. So first load balancer makes sure that the load is balanced among various app engines. Then compute engine performs data computation and publication of data. Multiple instances of compute engine are available to insure reliability and scalability. The data is stored and backed up using cloud storage. Big query allows speedy insertion of data in tables of cloud database. The results can be presented to the end users by means of various analysis and visualisation technique.

Key features:

- Run on Google's infrastructure

- Scalability

- Compute, storage and services

- Higher performance

- Provided support if required

- Assurance of Google Grade security and compliance for your applications

- Environment safe cloud 


\subsection{IBM BlueMix:}

IBM BlueMix is a platform as a service (PaaS) cloud which is developed by IBM. It supports programing languages like java, php, Python, Node.js, Go and many more. Integrated DevOps allows to build, run, deploy as well as to manage applications over IBM BlueMix cloud. BlueMix platform is based on Cloud Foundry open technology. It runs on Soft- Layer infrastructure. [9] The IBM Internet of Things Foundation is powered by IBM's following leading products and services: IBM DataPower Gateway, IBM WebSphere Application Server Liberty Core, IBM Informix TimeSeries, IBM MessageSight, Cloudant and SoftLayer.

IoT foundation shown in Figure-3 combined with IBM BlueMix platform provides powerful application access to IoT data and devices. IBM BlueMix support rapid development of analytics applications, visualization dashboard, and mobile IoT applications. You can create your IoT application with IBM Bluemix and then IBM provide REST and secure API to connect your device data with your application. IBM IoT foundation is the hub where you can set up and manage your connected devices. IBM IoT foundation uses MQTT protocol to securely transfer device data to cloud. [10]

Key features:

- Powerful web dashboard

- Device Registration

- Scalable connectivity

- Security of communication

- Storage of data

- Provided support if required

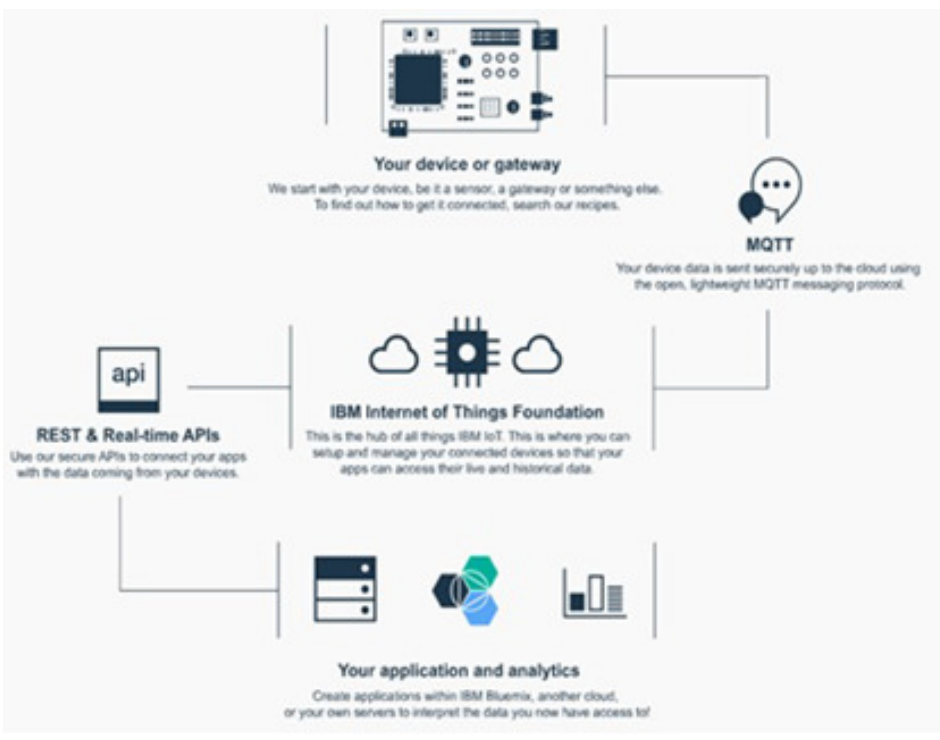

Figure 3. IBM IoT Foundation [10]

\subsection{ThingWorx}

ThingWorx is the first software platform designed to build and run the connected world applications. ThingWorx focuses on reducing the time, cost, and risk required to build innovative Machine-to-Machine (M2M) and Internet of Things (IoT) applications. ThingWorx accelerates 
IoT application development by compressing the design-develop-deploy cycle and reducing time to market. ThingWorx lets you deploy exactly the way you want either from cloud, on premise, federated or embedded, to fit the needs of any scenario. ThingWorx supports rapid creation of smart applications like: Smart Agriculture, Smart Cities, Smart Grid, Smart Water, Smart Buildings, and Telematics. It is a development suite that enables complete application design, runtime, and intelligence environment. [11] The ThingWorx Internet of Things Foundation is powered by a ThingWorx's following leading products and services: ThingWorx Composer, Codeless Mashup Builder, Event-Driven Execution and 3D Storage, ThingWorx SQUEAL, ThingWorx Edge MicroServer.

As shown in Figure-4 ThingWorx IoT platform provides device cloud to connect millions of devices with IoT application. It provides always on communication using REST, MQTT and sockets. Above the layer of communication there are system service integration, 3D storage engine and business logic. System service integration interact with business systems like ERP, CRM etc. 3D storage engine enables big data analytics. Above it there is a layer for REST APIs that helps to implement and use social services and cloud services. Then the data is presented via various visualization techniques.

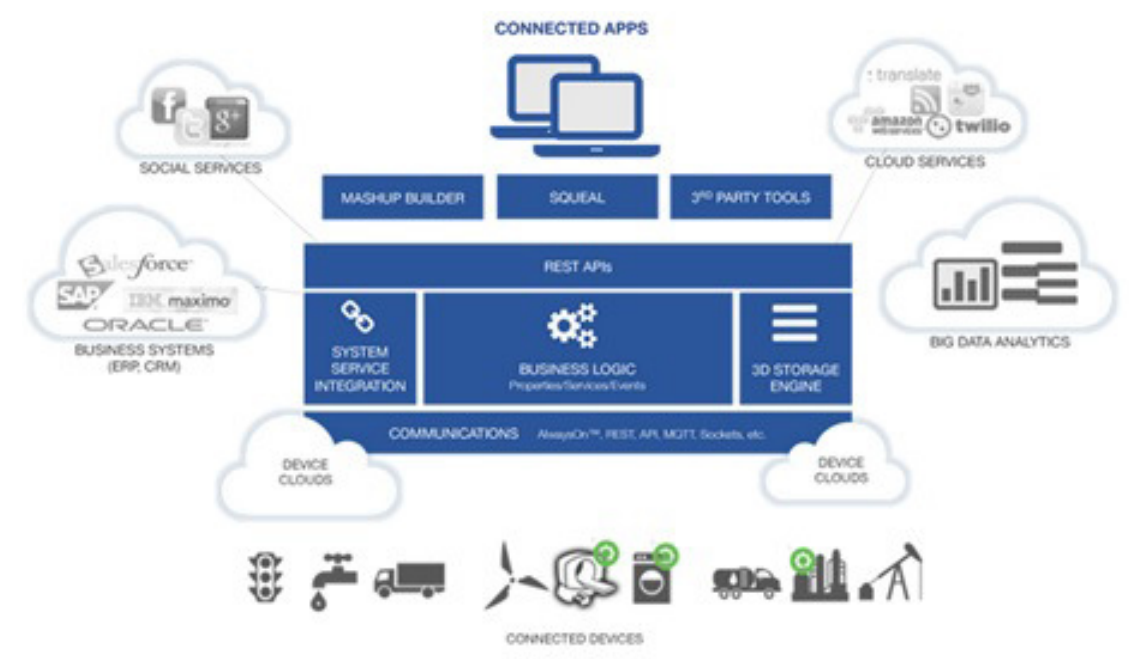

Figure 4. ThingWorx IoT foundation [11]

Key features:

- Modern and Complete Platform provisioning

- Faster deployment

- Integrate People, Systems and Machines

- Deploy in the way You Like to do it

- Let your Application evolve and grow Over Time

- Search-based Intelligence

- Allows collaboration

- Flexible Connectivity Options

\subsection{Microsoft Azure Cloud:}

According to Microsoft, Internet of Things for your business starts with the things that are involved in your business and affect it the most. It starts from the infrastructure of your business by adding devices and services into it with technological expansion, will lead you to get insight of the information and make powerful and informative business decision. Thus, here IoT is actually 
Internet of Your things. The piece of data about customers, sales, business processes or other inventory data is valuable asset to your organisation and it can help you to power your business.[12] Microsoft has the ability to transform your current business to a flexible intelligent system on your existing investment to achieve true flexibility. Microsoft provides Microsoft Azure Intelligent System Service which forms an integrated platform and services that builds Internet of Things systems and applications by gathering, storing and processing data. Intelligent systems services build upon Microsoft Azure helps organizations to securely connect, manage, capture and transform machine generated data into valuable information. Power BI, Office 365 and HD insight are powerful Microsoft assets that can be used to produce meaningful insights. Intelligent Systems Service provides agents and open-source agent software which help to support heterogeneous operating systems and protocols for Internet of Things System. With the help of Microsoft Cloud Compute facility, scalable data collection, processing and analysis can be done for your business processes. Cloud provides solution for data storage, data processing, data consumption and data analysis on real time or latent data. [13]

Figure-5 shows how Microsoft provides IoT solution. The core of Microsoft IoT foundation is Microsoft Azure cloud platform. It provides connectivity of millions of devices and sensors with IoT application. It provides remote access, monitoring, and content distribution and configuration management facilities for connected devices. It provides big data analysis, social as well as business integration and dash boarding tools to IoT application to build an IoT solution.

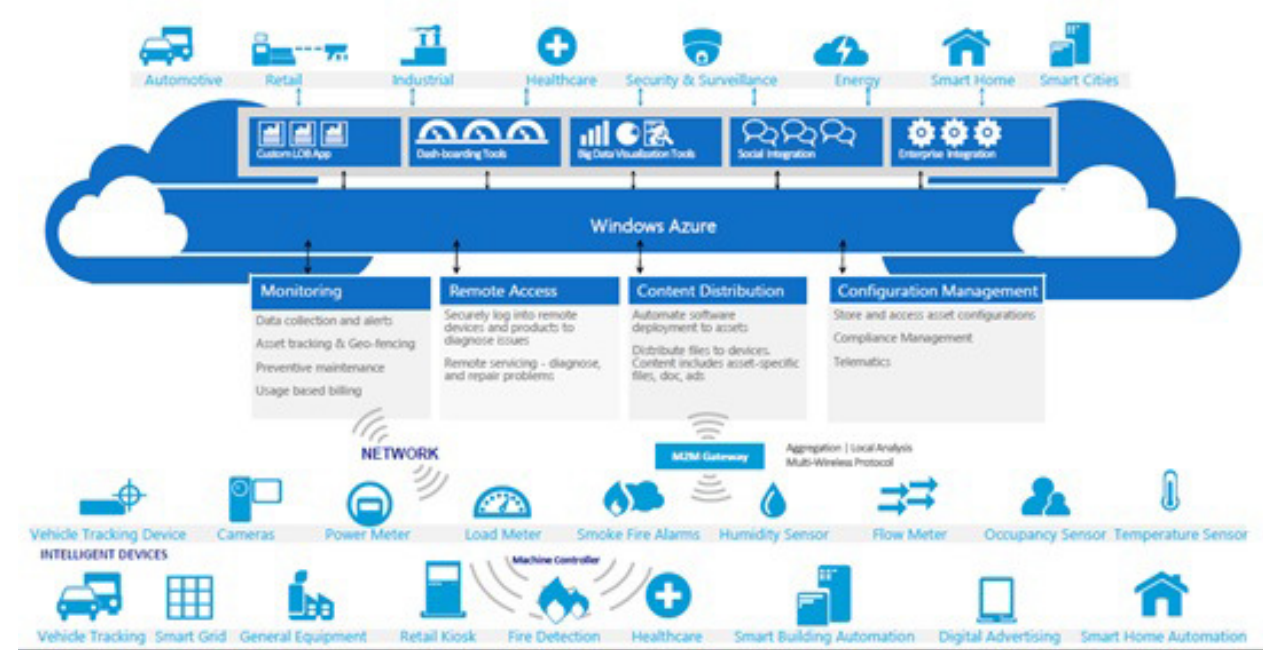

Figure 5. Microsoft IoT foundation [12]

Key features: [14]

- Build on what already established

- Get more benefits from your existing assets

- Small changes and big reflection

- Trusted support

- Expertise in development to deployment

- Connectivity of any device

- Skilled partners and powerful innovation

- Data insights

- Scalability

- Easy way for business transformation

- Agility 


\subsection{ThingSpeak}

ThingSpeak is an application platform for the development of IoT systems. It can help you to build the application which works upon the data collected by sensors. ThingSpeak is an open data platform for IoT application development. ThingSpeak is the perfect complement to an existing enterprise system to tap into the Internet of Things. It provides the ability to integrate your data with a variety of third-party platforms, systems and technologies, including other leading IoT platforms such as ioBridge and Arduino. [15] ThingsSpeak channel is used to send and store data. Each channel has: Eight fields that can hold any type of data, three location fields, and one status field. After creating a ThingSpeak channel, one can publish data to the channel, the data can be processed and application can retrieve the data. ThingSpeak platform provides following functionality to support IoT system: 1) Collect: Sends sensor and device data collected from it to the cloud so that the data can be further analysed. 2) Analyse: ThingSpeak can analyse the data received from sensors or devices and can derive the virtual representation of the data. 3) Act: Based upon the analysis, it will trigger the action to enable functioning of IoT system and application.

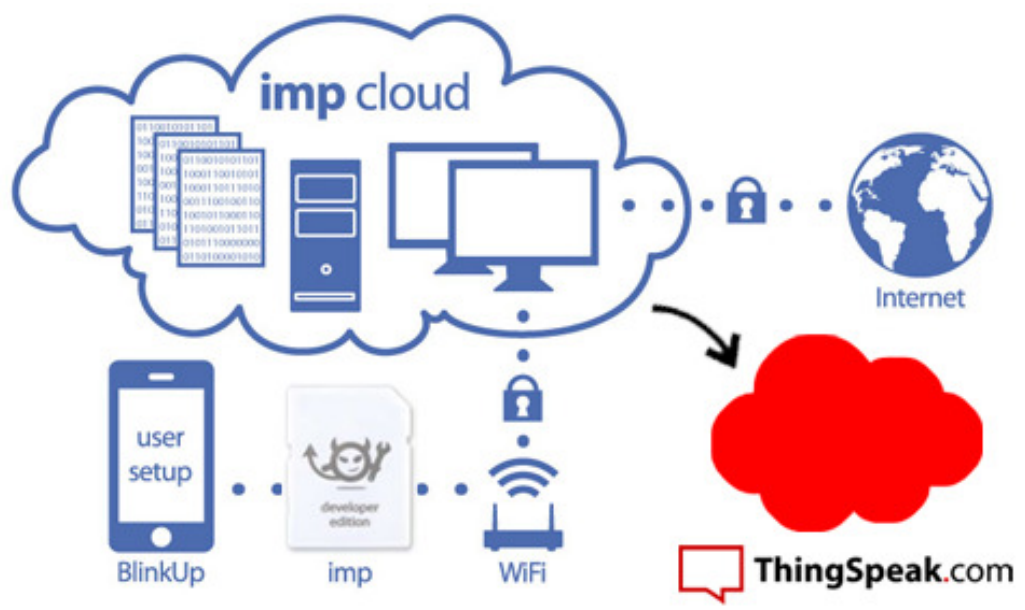

Figure 6. Electric Imp and ThingSpeak IoT [16]

Figure-6 shows ThingSpeak IoT with Electric Imp platform. Here Electric Imp is a platform with provides connectivity of Wi-Fi devices with cloud services. It provides access to the Electric Imp modules. Once the connectivity to such modules is simplified by Electric Imp cloud, data services from ThingSpeak are used to get IoT experience.

Key features:

- It provides real time collection of data storage

- Data analytics and Visualization using MATLAB

- Device Communication

- Open API support

- Provides Geolocation data

- Facilitates plugins

\subsection{Digital Service Cloud:}

Digital service cloud (DSC) is an open IoT platform. It allows IoT innovators to own their customers the way customers own their products. It supports product start-ups, global technological brands and product innovators. It is a platform that accelerates time to market 
process for a new innovation. By using the readymade infrastructure provided by Digital Service Cloud, one can build needed customised IoT solutions by connecting devices and using plug and play dashboard. Then one has to monitor and manage the product over its lifetime. It connects your product with a network of millions of devices. It runs a UI-driven rules engine that requires no coding. It monitors and streams a petabyte of real time data. It operates on a secure tenant based system and provides quick launch of your application with a wizard based app builders. [17]

Figure-7 shows DSC IoT foundation. Here consumer devices equipped with variety of sensors can connect to IoT application available on cloud and can communicate with application. Various networking and connectivity techniques are available that can connect the devices over cloud. Cloud provides device management and app development. Data analytics and visualisation through powerful dashboard are supported to end users.

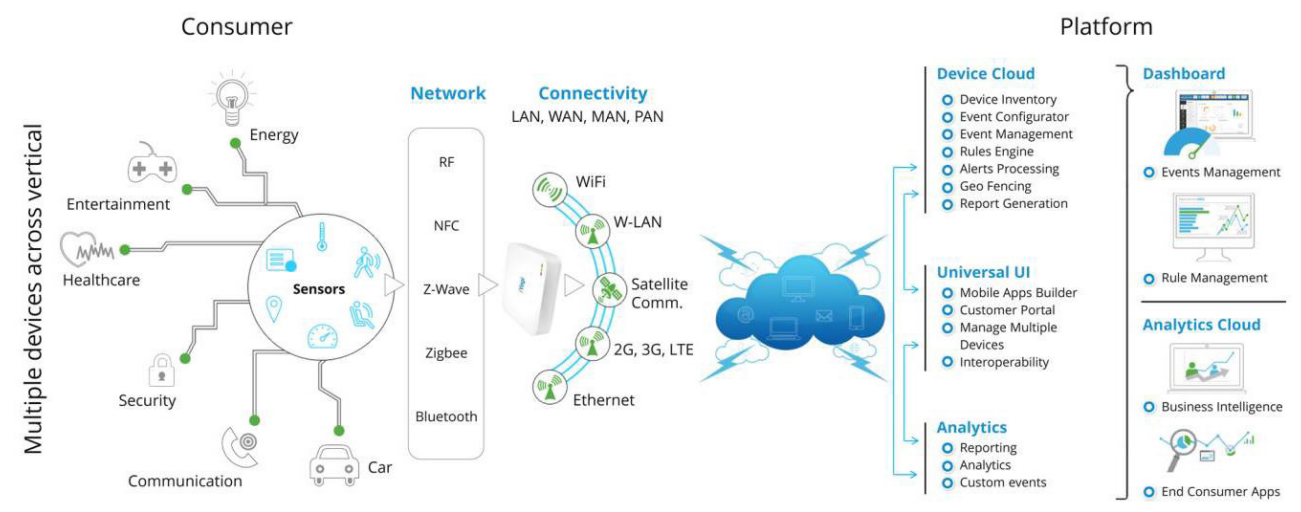

Figure 7. Digital Service Cloud IoT foundation [18]

Key features:

- Scalability

- Security

- Plug and Play

- Unified customer service

- Big data analytics

- Monitoring, maintenance and management of devices

- Multi-channel support delivery

- Preventive support through support cloud

- Users across diverse verticals

\subsection{Zetta:}

Zetta is an open source platform. It is developed in Node.js for creating IoT servers that run across geo-distributed servers and cloud. Zetta combines REST API, reactive programming and web sockets. This combination is suitable for assembling many devices into data intensive and real time applications. Zetta runs everywhere: It means zetta runs in the cloud, on PCs and on single board computers. Raspberry Pis, BwangleBones and PCs together can be linked with cloud platform like Heroku with the help of Zetta so that it can help to create geo-distributed networks. Zetta has the ability to turn any device into an API. By communicating with microcontrollers like Arduino and Spark Core, Zetta can provide every device a REST API as locally and also in the 
cloud. It provides support for assembling distributed system of devices that communicate and react via APIs. Zetta is a very developer friendly. It gives direct access of underlying protocols and conventions to developers so that they can easily and efficiently transform sensors, actuators and controllers into innovative IoT applications and systems. Architecture of Zetta is optimized for data intensive and real-time applications. Zetta allows monitoring of device and system behaviour in code and using visualization tools to get actionable insights. Also provides streaming of data into machine analytics platforms like Splunk. IoT projects consist of multiple devices across multiple locations running multiple applications developed by various companies. So, Zetta allows you to assemble smartphone apps, device apps and cloud apps together into large and complex systems. Example of such complex and large scale systems is home automation, smart transportation and wearable computing. [19]

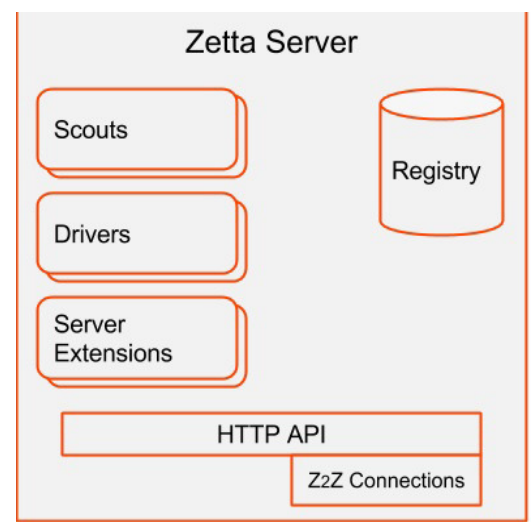

Figure 8. Zetta Server architecture [20]

Figure-8 shows Zetta server architecture. Zetta server which is at highest level of abstraction contains elements like drivers, scouts, server extension, apps etc. It runs on a hardware hub like raspberry Pi and allows components to communicate with devices. It then produces API to the consumer. Here Scout is a device discovery technique over network. One Zetta server will reside on hardware hub and another on cloud. Server on hardware will contact to that in the cloud. So Zetta will provide API to consumers.

Key features:

- Run everywhere

- Provides API to everything

- Provides developer friendly environment

- Streaming of big data along with and data insights

- Development support of large applications for efficient development

\subsection{Nimbits:}

Nimbits is a PaaS that can be downloaded on any from Raspberry Pi, Web Server, Amazon EC2, or Google App Engine. The platform is used for developing hardware and software solutions that can connect to the cloud or to each other and enables logging and retrieving large amounts of data from physical devices, triggering events or alerts, or performance of complex analysis.[21] Nimbits is a platform for connecting people, sensors and software to the cloud and with one another. It is based upon data logging and rule based technology. It resolves the complexity associated with Edge Computing in IoT by facilitating a platform which is built upon embedded system locally and then filtering noise, running rule engine and then pushing data that are very important on the cloud. It first records and then processes geo informatics and time stamped data 
and then produces rule form that information. Here rules can be email alerts, push notification, statistics or any calculation. Nimbits Public Cloud is an instance of Nimbits server.

Key features:

- Download Nimbits servers on chips, servers or on the cloud

- It is an Open-source platform

- Facilitates event triggers and alerts

- Process geo and time-stamped data

- Build provided for Google App Engine and Linux Systems for development

- Compatible with most J2EE servers

\subsection{Yaler:}

Yaler is a pay-as-you use platform which provides a relay infrastructure which offers secure access to embedded systems and it works with any device with a TCP socket. Yaler is a costeffective solution which is suitable for enterprise applications. It also offers a hosted service on dedicated relay instances. A fixed yearly fee per relay instance helps you to max out the number of devices connected to a dedicated instance. The Yaler relay service supports SSL/TLS encryption. The integration of Yaler.net, a cloud-based connectivity service, enabled a secure adhoc connection for data streaming. [22] It is mainly designed to provide a stable, secure, and high performance execution environment for applications running on Amazon EC2. [23]

Key features:

- It provides plug-and-play functionality for end users

- Access via any browser or even phone

- Enables addressability and accessibility for devices blocked by firewalls, NAT or mobile routers

- It provides access to your device from a client such as a browser, Android, etc.

\subsection{Amazon Web Services:}

Amazon Web Services (AWS) allows Internet of Things on a global scale by facilitating security, services and support. It facilitates immediate access to desired computing power by means of Amazon Elastic Cloud Compute (EC2). Helps to performs big data analytics and supports high volume data. Amazon Kinesis helps to ingest data from thousands of sensors. AWS provides security to your data which can be in transit or at rest. AWS provide pay-as-you-go model for IoT applications. It has it multiple pricing models like tiered pricing, Reserved Instances, and an active marketplace. AWS supports on demand infrastructure to accommodate need of IoT system. It provides access to more storage, compute capability and global resources when needed. AWS provides flexibility for IoT applications in terms of tools, programing languages, data management and other infrastructure resources. ActiveMq and Mosquitto servers help in managing and analysing IoT applications. User identity, device analytics, and device messaging/notifications are all common parts of an Internet of Things application. Amazon Web Services provides services that take the effort out of these important parts of your application. Services like Amazon Cognito, Amazon Mobile Analytics, and Mobile Push to take care of the undifferentiated heavy lifting while you focus on the differentiated benefits of application. [24]

As shown in Figure-9, Amazon Kinesis can collect high throughput data from devices and gateways, and then it can analyze and store it over cloud so that applications can consume it and can generate quick decision. It can support data up to any scale. 


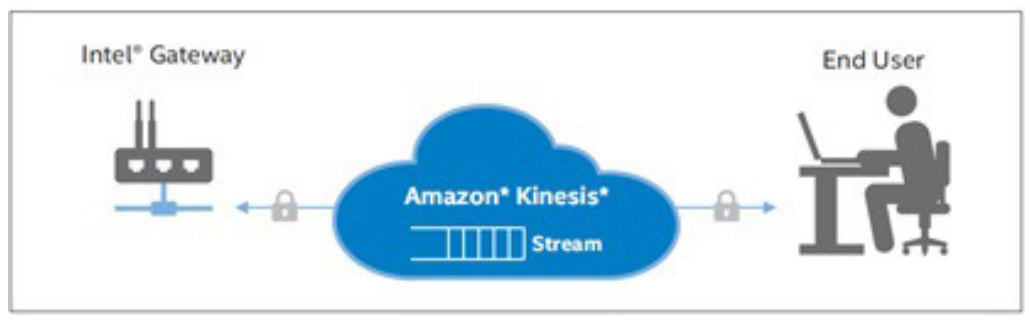

Figure 9. Data streaming from Gateway to cloud by Amazon Kinesis [25]

Key features:

- Scalability

- Privacy and Security

- Pay-as-you go model

- High availability and flexibility

- Data analytics and storage of high volume data

- On demand services like other platforms

- Provides Hardware resources

\subsection{Axeda and Oracle Java Embedded:}

The Axeda Machine Cloud service provides M2M services, IoT connectivity services, software agents and toolkits that allow you to select communication method and hardware which is appropriate for your IoT solution. [26] The Axeda IoT platform provides end-to-end enterprise capabilities using Java EE technology and the Oracle Database. It gives facilities like message queuing for reliable end-to-end data transfer, security based on SSL communication with authentication and authorization, device and asset management, tracking and monitoring, complex event processing, location services, and more. Oracle's Java Embedded solutions aim to support massive amounts of data required for and created as a result of the Internet of Things by facilitating seamless communications between all elements of the IoT architecture. It delivers an integrated, secure and comprehensive platform for the entire IoT architecture across all vertical markets. Oracle enables real-time response and data capture from millions of device endpoints. Oracle offers several solutions, including Oracle Java SE Embedded, Oracle Java ME Embedded, Oracle Java Embedded Suite and Oracle Event Processing for Oracle Java Embedded to meet your specific technology requirements. [27]

Figure-10 shows Axeda IoT platform stack. IoT connectivity is at bottom level. Above it IoT data management layer is there to manage data coming from devices. Device and assets are managed by using cloud platform. At the top level application service and integration framework support application development and deployment. Device and asset configuration management is also supported by cloud.

Key features:

- Real time data capture

- Millions of device

- It is an M2M platform with proven middleware capabilities

- Also facilitate communications among devices 
Table 1. Comparison of various IoT platforms

\begin{tabular}{|c|c|c|c|c|c|c|c|c|c|c|c|}
\hline & $\begin{array}{l}\text { Google } \\
\text { Cloud } \\
\text { Com- } \\
\text { pute }\end{array}$ & $\begin{array}{l}\text { IBM } \\
\text { Blue } \\
\text { Mix }\end{array}$ & $\begin{array}{l}\text { Thing- } \\
\text { Worx }\end{array}$ & $\begin{array}{l}\text { Micro- } \\
\text { soft } \\
\text { Azure }\end{array}$ & $\begin{array}{l}\text { Thing } \\
\text { Speak }\end{array}$ & $\begin{array}{l}\text { Digi- } \\
\text { tal } \\
\text { Ser- } \\
\text { vice } \\
\text { Cloud }\end{array}$ & Zetta & Nimbits & Yaler & $\begin{array}{l}\text { Amazon } \\
\text { Web } \\
\text { Service }\end{array}$ & $\begin{array}{l}\text { Axeda } \\
\text { Oracle } \\
\text { Java } \\
\text { Embe- } \\
\text { dded }\end{array}$ \\
\hline Scalability & $\sqrt{ }$ & $\sqrt{ }$ & $\sqrt{ }$ & $\sqrt{ }$ & $\sqrt{ }$ & $\sqrt{ }$ & $x$ & $\sqrt{ }$ & $\sqrt{ }$ & $\sqrt{ }$ & $\sqrt{ }$ \\
\hline $\begin{array}{l}24 * 7 \\
\text { availability }\end{array}$ & $\sqrt{ }$ & $\sqrt{ }$ & $\sqrt{ }$ & $\sqrt{ }$ & $\sqrt{ }$ & $\sqrt{ }$ & $\sqrt{ }$ & $\sqrt{ }$ & $\sqrt{ }$ & $\sqrt{ }$ & $\sqrt{ }$ \\
\hline $\begin{array}{l}\text { Security } \\
\text { and } \\
\text { Privacy } \\
\text { provisi- } \\
\text { oning }\end{array}$ & $\sqrt{ }$ & $\sqrt{ }$ & $\sqrt{ }$ & $\sqrt{ }$ & $x$ & $\sqrt{ }$ & $x$ & $x$ & $x$ & $\sqrt{ }$ & $\sqrt{ }$ \\
\hline $\begin{array}{l}\text { Plug and } \\
\text { Play }\end{array}$ & $\sqrt{2}$ & $\sqrt{ }$ & $\sqrt{2}$ & $\sqrt{ }$ & $x$ & $\sqrt{ }$ & $x$ & $x$ & $x$ & $\sqrt{ }$ & $\sqrt{2}$ \\
\hline $\begin{array}{l}\text { Support } \\
\text { for } \\
\text { millions of } \\
\text { devices }\end{array}$ & $\sqrt{ }$ & $\sqrt{ }$ & $\sqrt{ }$ & $\sqrt{ }$ & $x$ & $\sqrt{ }$ & $x$ & $x$ & $x$ & $\sqrt{ }$ & $\sqrt{ }$ \\
\hline $\begin{array}{l}\text { Real time } \\
\text { data }\end{array}$ & $\sqrt{ }$ & $\sqrt{ }$ & $\sqrt{ }$ & $\sqrt{ }$ & $\sqrt{ }$ & $\sqrt{ }$ & $x$ & $\sqrt{ }$ & $x$ & $\sqrt{ }$ & $\sqrt{ }$ \\
\hline $\begin{array}{l}\text { Storage of } \\
\text { data }\end{array}$ & $\sqrt{2}$ & $\sqrt{ }$ & $\sqrt{2}$ & $\sqrt{ }$ & $x$ & $x$ & $x$ & $\sqrt{1}$ & $x$ & $\sqrt{ }$ & $\sqrt{2}$ \\
\hline $\begin{array}{l}\text { Provision } \\
\text { of } \\
\text { Support }\end{array}$ & $\sqrt{ }$ & $\sqrt{ }$ & $\sqrt{ }$ & $\sqrt{ }$ & $\sqrt{ }$ & $\sqrt{ }$ & $x$ & $x$ & $\sqrt{ }$ & $\sqrt{ }$ & $\sqrt{ }$ \\
\hline $\begin{array}{l}\text { Developer } \\
\text { Friendly }\end{array}$ & $\mathrm{H}$ & $\mathrm{H}$ & $\mathrm{M}$ & $\mathrm{H}$ & $\mathrm{M}$ & $\mathrm{M}$ & $\overline{\mathrm{H}}$ & $\overline{\mathrm{L}}$ & $\mathrm{H}$ & $\mathrm{M}$ & $\mathrm{H}$ \\
\hline $\begin{array}{l}\text { Solution } \\
\text { Type }\end{array}$ & PaaS & PaaS & $\begin{array}{l}\text { Comp- } \\
\text { lete } \\
\text { Io T }\end{array}$ & PaaS & $\begin{array}{l}\text { Data } \\
\text { Ana- } \\
\text { lytic }\end{array}$ & PaaS & $\begin{array}{l}\text { API } \\
\text { to } \\
\text { De- } \\
\text { vices }\end{array}$ & PaaS & Left & IaaS & SaaS \\
\hline $\begin{array}{l}\text { Solution } \\
\text { Type }\end{array}$ & F.T & F.T & F.T & F.T & 0.5 & 0.5 & $0 . S$ & 0.5 & F.T & F.T & 0.5 \\
\hline
\end{tabular}

\section{COMPARISON}

The comparison table TABLE.1 here shows comparison for various parameters among all discussed platforms. The abbreviations taken in this table are: H-High, M-Moderate, and L-Low. Various parameters used for comparison are like developer friendliness, scalability, availability, solution type, security and privacy, open source/ free trial, plug and play, support for millions of devices, support for real time data, data storage, support provisioning etc.

\section{CONCLUSiON}

From the content of this paper, now we can conclude that though all the discussed platforms are very well known for IoT solution development, ThingWorx and Microsoft Azure are the most promising platforms for IoT solution compare to the others. Google Cloud platform is popular for its compute engine, app engine and container engine. IBM BlueMix is popular for its powerful app creation and management support, 3rd party API and services that it provides to its users. ThingWorx IoT platform is popular for its overall IoT solution creation as well as support for 3rd party device cloud facility, open API and Always On features. Microsoft Azure cloud is different 
from others for its networking capabilities as well as compute capabilities. ThingSpeak is mainly intended to provide data analytics and visualisation. Zetta platform provides API to all devices. Yaler is popular for its facility of relay infrastructure for applications running on Amazon EC2. Amazon web service is popular for its IaaS facility with higher scalability. At the last Axeda Oracle Java Embedded IoT platform is popular for its predictive maintenance, usage based insurance and warranty billing.

\section{ACKNOWLEDGEMENTS}

The author would like to thank Prof. T.A.Champaneria for his continuous guidance and encouragement through the work.

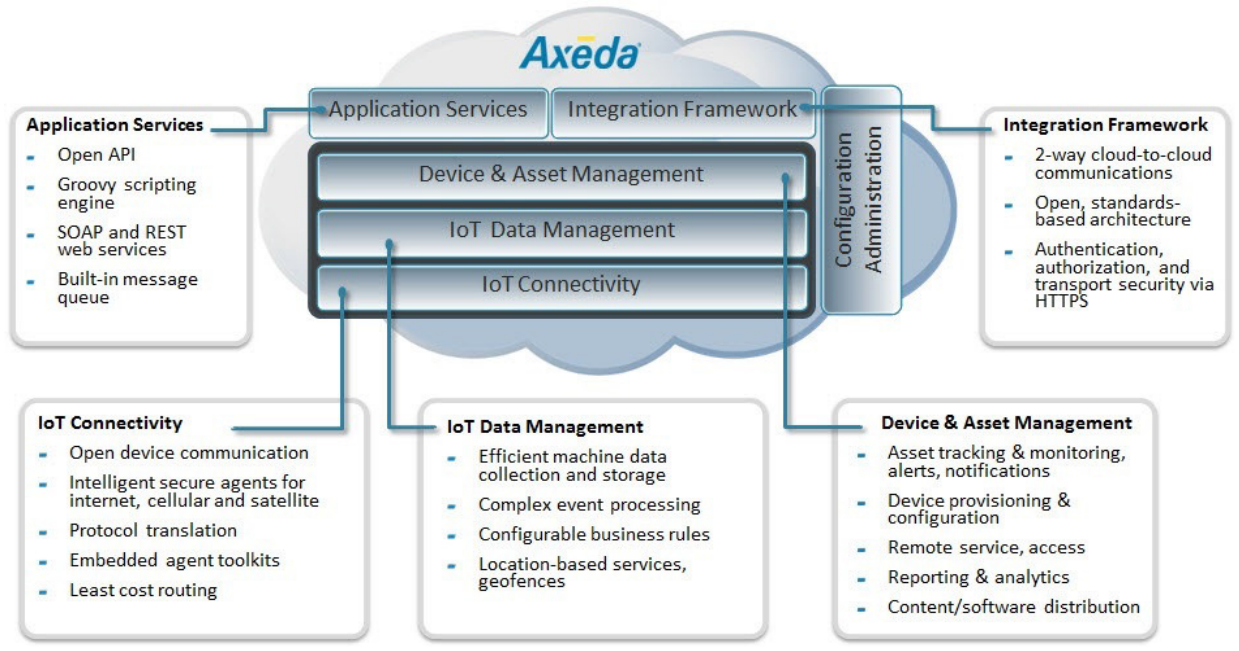

Figure 10. Axeda IoT stack [28]

\section{REFERENCES}

[1] L. Coetzee and J. Eksteen, "The Internet of Things Promise for the Future an Introduction," ISTAfrica Conference Proceedings, pp. 1- 9, 2011.

[2] https://www.arm.com/markets/internet-of-things-iot.php

[3] http://technologicalthemes.blogspot.in/2009/09/ differenciating-between-platform.html

[4] "Platforms for the Internet of Things an Analysis of Existing Solutions."- A university paper.

[5] http://www.zatar.com/blog/what-is-an-iot-application-platform

[6] http://www.digitalservicecloud.com/iot-middleware.html

[7] https://cloud.google.com/solutions/iot/

[8] https://cloud.google.com/solutions/architecture/streamprocessing

[9] https://en.wikipedia.org/wiki/Bluemix

[10] https://internetofthings.ibmcloud.com

[11] http://www.thingworx.com/iot-platform

[12] B. Edson, "Creating the Internet of Your Things", Microsoft Corporation

[13] J. Shewchuk, "Internet of Things", Microsoft Corporation

[14] "Ten reasons your business needs a strategy to capitalize on the Internet of Things today," 2015, Microsoft Corporation

[15] https://thingspeak.com

[16] http://community.thingspeak.com/tutorials/electric- imp/data-storage-and-retrieval-with-thingspeak/

[17] http://www.digitalservicecloud.com

[18] “The Best Platform for IoT Innovators", 2015, iYogi

[19] http://www.zettajs.org 
International Journal of Computer Science \& Engineering Survey (IJCSES) Vol.6, No.6, December 2015

[20] http://www.zettajs.org/reference/2014/12/17/Zetta-Overview.html

[21] http://www.nimbits.com/index.jsp

[22] http://blog.yaler.net

[23] https://yaler.net/

[24] https://aws.amazon.com

[25] A. Kinesis, E. User, and A. Kinesis, "Scaling Internet of Things Data Movement with Amazon * Kinesis *," no. November 2014.

[26] https://blog.profitbricks.com/top-49-tools-internet-of-things/

[27] http://www.ptc.com/axeda/product/iot-connectivity

[28] E. J. Bruno, J. Kornwitz, P. Lombardi, and M. Samuelsson, "The Intelligent Internet of Things with Axeda and Oracle Java Embedded," no. June, 2014.

\section{Authors}

Bhumi Nakhuva is a final year M.E student at L. D. College of Engineering, Ahmedabad under the affiliation of Gujarat Technological University. She is pursuing her M.E. dissertation in the area of IoT under the guidance of Prof. Tushar Champaneria.

Prof. Tushar A Champaneria is an Assistant professor in Computer Engineering department at L. D. College of Engineering, Ahmedabad. He has completed M.E (CSE) from BITSPilani. He is pursuing his $\mathrm{PhD}$ in the area of IoT.

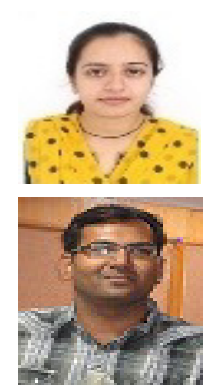

